\title{
Self-assembly of copolymers and lipids
}

\author{
Xiao-jun Li, M.Schick \\ Department of Physics, Box 351560 , \\ University of Washington, Seattle 98195-1560, USA
}

Received August 14, 2000

Recent progress is reviewed in the understanding, and computation, of self-assembly in block copolymers and in biological lipids, both neutral and charged.

Key words: self-assembly, lyotropic phases, block copolymers, lipids

PACS: $61.25 . \mathrm{Hq}, 87.15 .-\mathrm{v}, 87.30 . \mathrm{Hq}$

\section{Block copolymers}

Block copolymers self assemble into a great array of stable, ordered, phases in which components of different blocks are separated. The basic reason for this is clear. The monomers comprising the different blocks prefer energetically to be surrounded by monomers of the same kind and to avoid contact with monomers of other species. At sufficiently low temperatures, therefore, the polymers will arrange themselves so as to minimize contact between the different species of monomer. Were the blocks not chemically bonded to one another, they would undergo phase separation. Because they are so bonded, they cannot separate but must arrange themselves in an ordered manner which minimizes these contacts subject to various constraints. One of these constraints is that the system be incompressible, or at least nearly so. The simplest ordered phase is lamellar, in which the lamellae are composed of a majority of one monomer or the other. In the case of diblock copolymers consisting of blocks of $A$ and blocks of $B$ monomers, there is a lamellae composed of a majority of $A$ followed by one of a majority of $B$, then $A$ again and so forth. At very low temperatures in which the monomers are well separated, called the strong segregation limit, the interfaces between the different lamellae are quite narrow, much narrower than the width of the lamellae themselves. Most of the energy of the system arises from the contact between monomers at these internal interfaces between lamellae. Because simplicity is introduced by the assumption of very low temperatures, much theoretical progress has been made in this limit [1].

At sufficiently high temperatures, of course, the system is disordered due to the dominance of entropy considerations, but will order when the temperature is reduced 
sufficiently. The transition temperature is, to a good approximation, determined by the product $\chi N$ where $k T \chi$ is the characteristic energy difference between the interactions of an $A$ monomer with its fellows and with a $B$ monomer, and $N$ is the number of statistically independent units in the block. Thus $\chi^{*} N=$ constant determines the transition temperature $T^{*}$. Note that the transition temperature is proportional to the length of the blocks, because longer blocks have a much smaller entropy per monomer, making it easier for the system to order. When the system is near the transition temperature, progress can also be made. The order parameter, which is a measure of the difference in the local density of $A$ and $B$ monomers, is small presumably, so that one can hope to approximate the free energy in a Landau expansion, and to include fluctuations to Gaussian order; i.e. by means of the random phase approximation [2]. In this limit, the lamellae are not well ordered, i.e. there is not much separation of the monomers; hence this regime is denoted the weak segregation limit. The width of the internal interfaces is very wide, as large as that of the lamellae themselves. The energy comes from the presence of monomers of one kind in lamellae in which the other monomer is dominant, however weakly. The contribution of the entropy to the free energy is as important as that of the energy.

There are many ordered phases other than the lamellar one which occur in these systems, and which has the lowest free energy depends primarily on the architecture of the polymer, and on the temperature of the system. By "architecture" is meant the relative size of the different blocks. Again consider the case of the diblock copolymer. If the sizes of the two blocks are comparable, then one expects the lamellar phase to be most stable. But if one block is significantly smaller than the other, the system chooses a phase in which the larger entropy of the larger blocks can be expressed at the expense of the smaller entropy of the shorter blocks, again subject to constraints of incompressibility. Thus in the commonly observed hexagonal phase, characterized by cylinders of the minority component packed in a hexagonal array with the majority component between them, the latter enjoys the larger entropy associated with the space outside the cylinders while the minority component has its entropy reduced as the chains must arrange themselves inside the confines of the cylinders. As the imbalance between the lengths of the blocks increases, the cylinders are replaced by spheres, and the hexagonal phase gives way to one in which the spheres are arranged in a body centered cubic packing.

All of this was well known and could be described by both weak and strong segregation theories. However there were several other phases which had been observed, reported to be stable, and yet had not been predicted by either theory. Such phases included the double-diamond phase $(\operatorname{Pn} \overline{3} \mathrm{~m})$ [3], the perforated lamellar phase [4], and the gyroid phase $(\operatorname{Ia} \overline{3} \mathrm{~d})$ [5]. This was an odd situation in that the self consistent field theory description of polymers due to Edwards [6] and extended to block copolymers by Helfand and coworkers [7], should have been a very good description of such systems. Indeed the strong segregation theory and the Landau expansion of the weak segregation theory were simply approximations to it. Further the self consistent field theory had been successfully applied to numerous problems. 
The difficulty was resolved in 1994 [8]. In retrospect, there was no problem with the self-consistent field theory itself. However it had always been implemented in real space, and a real space description of such phases as the double diamond or gyroid was so difficult that it had not even been attempted. In contrast to such a procedure, Matsen and Schick expanded all functions of position, such as the monomer density, into a complete set of functions which had the space group symmetry of the phase to be described. This converted the self-consistent equations for the position-dependent functions into an infinite set of equations for the coefficients in these expansions. The equations were then solved numerically, and the free energy of the phase with given symmetry obtained. By comparison of the free energies of phases with different symmetries, the phase diagram was established.

There were several notable results. First, of all the non-standard phases which had been reported, only the gyroid phase was found to be stable, and found precisely where it had been observed [5]. Second the double diamond phase was found not to be a globally stable phase, and rather far from being the phase with lowest free energy. Third the perforated lamellar phase was found to be metastable, but not the phase of lowest free energy. These predictions caused these phases to be reconsidered experimentally. It was found that the double diamond phase had been misidentified, and was in reality a gyroid phase [9]. It was also found that the perforated lamellar phase was indeed metastable [10].

The method employed by Matsen and Schick essentially permitted the solution of the self-consistent field theory in full, without the necessity of resorting to additional approximations such as those employed in the strong and weak segregation theories. It permits the self consistent field theory to be solved over the whole range of experimentally accessible segregations, thus unifying the weak and strong segregation theories [11]. It also enabled an examination of the mechanisms behind the self assembly. Certainly minimization of the area of the internal interfaces, subject to the given volumes of the two blocks, is one of the important driving forces. This minimization, by itself, would predict that the internal interfaces would be surfaces of constant mean curvature [12]. But this is not the only constraint. That of incompressibility is equally important [13], and causes these surfaces not to be ones of constant curvature, a prediction that has been recently verified experimentally [14].

Other notable applications of the above theory are to thin copolymer films [15], ternary mixtures of $A$ homopolymer, $B$ homopolymer, and $A B$ diblock [16], and to tilt [17] and twist [18] grain boundaries within lamellar phases of diblock copolymers.

\section{Biological lipids}

Biological lipids display the same array of ordered phases as those of block copolymers. Given that these lipids are almost invariably found in the lamellar-like bilayer configuration of biological membranes, the question is whether their ability to adopt other configurations is of biological significance [19]. There is a particularly remarkable example in lipids with phosphatidylethanolamine (PE) headgroups, lipids which are a substantial constituent of membranes. Such lipids, by themselves, do not form 




Figure 1. Phase diagram of an anhydrous system of lipids as a function of the temperature $T_{N}^{*} \equiv 1 / 2 \chi N$ and the fraction $f$ of the total lipid volume which the headgroup occupies. In addition to the disordered (D) and lamellar $\mathrm{L}_{\alpha}$ phases, there are body-centered cubic (bcc), hexagonal $(\mathrm{H})$, and gyroid $(\mathrm{G})$ phases. The subscripts I and II denote normal and inverted phases respectively. The dashed lines indicate extrapolated boundaries.

lamellar phases under biological conditions, but rather hexagonal phases. They are in the bilayer configuration only because the other lipids comprising it prefer this configuration. What are the PE lipids doing in this membrane? A few answers to this question have been proposed. One that seems reasonable is that the presence of such lipids makes it easier to form non-bilayer configurations which are often needed by the membrane. For example, much of the transport of material within the cell takes place by the movement of vesicles which encounter one another, fuse and exchange contents, and move on. In the fusion process, one believes that there is some intermediate configuration which is not lamellar. Presumably the free energy of this intermediate would be reduced by the presence of PE lipids.

In order to investigate theoretically the role of non-lamellar forming lipids in biological membranes, one must have some model system of lipids which displays all the non-lamellar phases which have been observed. As the method described above for block copolymers is ideally suited to the description of phases of any space group symmetry, it is natural to apply it to the problem of self assembly of lipids as well. We have done that. We model lipids with two identical tails composed of $N$ units each, the units being characterized by their volume. The tails are treated as if they were flexible chains. The headgroup is characterized solely by its volume. Therefore the lipids are characterized by a single architectural parameter, denoted $f$, which is the volume of the headgroup relative to that of the entire lipid. As biological lipids are always in a water environment, we include a solvent as well, one also 


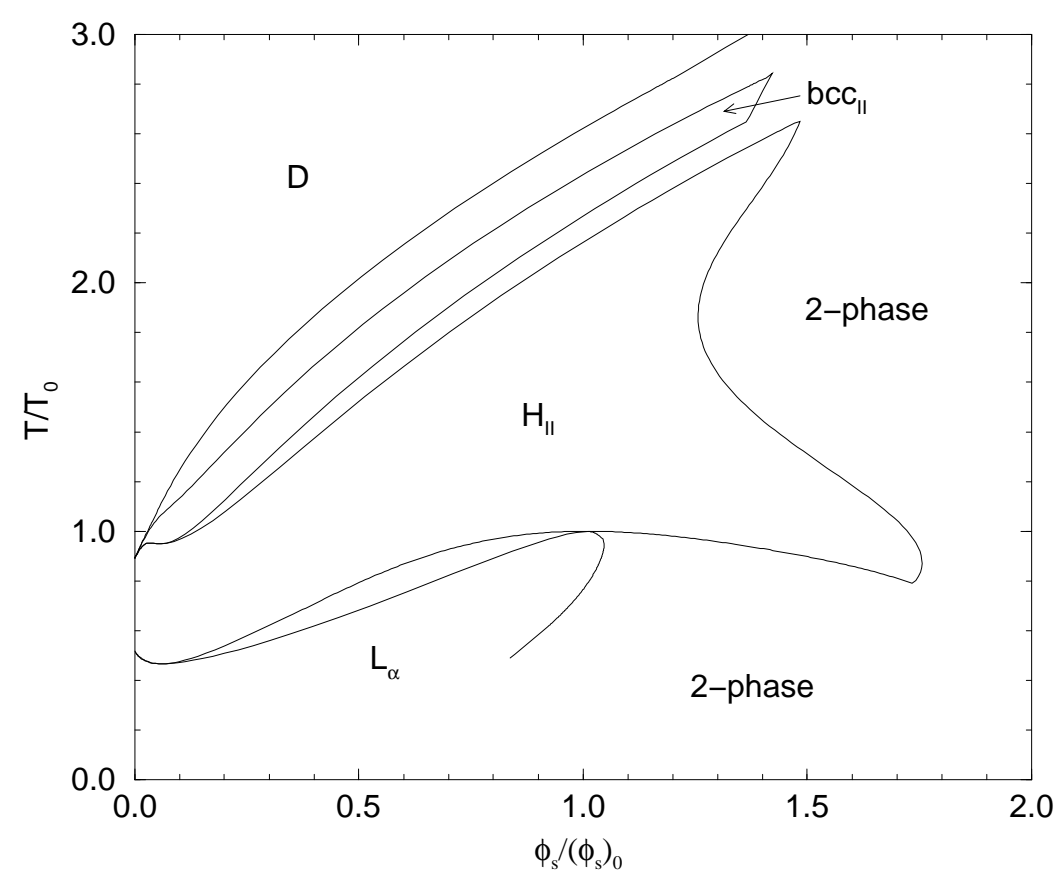

Figure 2. Phase diagram of a neutral lipid with $f=0.24$ in a solvent with volume that of water. The diagram is shown as a function of the temperature, $\mathrm{T}$, measured in units of $\mathrm{T}_{0}$, the temperature of the azeotrope between $\mathrm{L}_{\alpha}$ and $\mathrm{H}_{\|}$ phases and the volume fraction of solvent with respect to that at the azeotrope, $\phi_{s} /\left(\phi_{s}\right)_{0}$.

characterized solely by its volume. The interactions in the system are few. There is a repulsive interaction between the chains and the headgroup, and between the chains and solvent. For simplicity, both of these interactions are taken to be of the same strength, $k T \chi$. The incompressibility constraint is enforced, which models hard core repulsions. When the lipids to be modelled are charged, such as phosphatidylserine (PS), Coulomb interactions between headgroups and counter ions are included, as can an interaction between charges and the neutral solvent, one which models the charge, dipole interaction. Self-consistent field theory is employed.

We have calculated the phase diagram of our lipids under various conditions. First we considered their phase diagram in the absence of solvent, as a function of temperature and of the architectural parameter $f$. The result [20] is shown in figure 1. Second we calculated the phase diagram of a given lipid, i.e. given value of $f$, in a solvent as a function of solvent concentration and temperature. The result is shown in figure 2. Consider figure 1 first. The diagram shown here can be compared to experiment by noting that different values of $f$ correspond to different lipids with headgroups of different relative size. One sees that for small headgroups, the system assumes "inverted" phases, in which the headgroup plays the role analogous to that of the minority component in the diblock system. As the headgroup becomes larger, the lamellar phase is stabilized, and then other "normal" phases, with the headgroup playing the role analogous to the majority component. This phase diagram is a concrete realization of the idea of Israelachvili [21] in which the lipids are de- 
scribed as if they had a "shape"; cone-like, cylindrical, etc. Further, cone-like lipids form hexagonal phases, cylinders stack in bilayers etc. While this concept is useful in rationalizing various results, its predictive power is limited [22]. For example, it is difficult to understand how the shape of a lipid changes abruptly at a phase transition; the concept cannot be used to predict such transitions. In our model, we have no such problem. The lipid has one, unchanging, architectural parameter, and this lipid undergoes many phase transitions. For example, in figure 2 the phase diagram [20] is shown for a neutral lipid with an architectural parameter $f$ appropriate to that of dioleoylphosphatidylethanolamine (DOPE). The result is in good agreement with experiment [23], even reproducing the re-entrant inverted hexagonal phase.

With such a model of lipids, one can begin to address the effect of a non-lamellar forming lipid in a mixture with other lamellar forming lipids. This has been done in two papers. We would like to investigate the distribution of the different lipids in a fusion intermediate, perhaps something like a stalk [24]. In such a stalk, the lipids are in a non-uniform environment, and one wants to know whether the density of nonlamellar formers is increased in this environment. Not being able to produce such an intermediate yet, we looked at the non-uniform inverted hexagonal phase of a mixture of lamellar- and non-lamellar forming lipids [25]. We found that, indeed, the fraction of non-lamellar formers increased in the regions which were most difficult for the tails to fill, regions which would occur in a fusion intermediate. We were able to quantify this increase. In a third paper [26], we investigated the effect of fluctuations in such a system utilizing the random phase approximation [27]. This permitted us, inter alia, to calculate the structure function of the lamellar phase. We would like to determine the role of the non-lamellar formers in bringing about the change from the bilayer to a bilayer with a non-bilayer, non-uniform, fusion intermediate. Not being able yet to create a fusion intermediate, we could instead quantify the dominant role the non-lamellar former takes in bringing about the phase transition from lamellar phase to non-uniform hexagonal phase.

In the case of charged lipids, we reproduce [20] the inverted hexagonal to lamellar phase transition that PS undergoes as the $\mathrm{pH}$ is increased [28]. The way this phase transition comes about is as follows. The PS headgroup is sufficiently small that the neutral lipid prefers the inverted hexagonal phase. When the $\mathrm{pH}$ is increased, the headgroup becomes charged, and this charge attracts to the headgroups counterions enlarged by waters of hydration, such as $\mathrm{H}^{+} \cdot 4 \mathrm{H}_{2} \mathrm{O}$. These counterions effectively increase the size of the headgroups which, as we have seen, causes a transition from inverted hexagonal to a lamellar phase. Hafez et al. [29] have recently shown that one can tune the $\mathrm{pH}$ at which a vesicle containing both anionic and cationic lipids becomes unstable. We identify the instability as the same as that of the lamellar phase to the inverted hexagonal phase. The tunability of the $\mathrm{pH}$, of great utility for drug delivery, is easy to understand. Consider at a given $\mathrm{pH}$ a vesicle containing anionic lipids which prefer to form an inverted hexagonal phase. The vesicle is stabilized by the presence of counter ions near the headgroup. If cationic lipids are now added, the number of stabilizing counter ions is reduced. To restore the stability, more counter ions must be attracted to the headgroup. This is accomplished by in- 
creasing the charge on the headgroups, i.e. by increasing the $\mathrm{pH}$. Thus one expects the $\mathrm{pH}$ at which the instability occurs to be an increasing function of the density of the cationic lipid, as is observed [29].

One nice feature of our model is that it permits us to observe different effects of the Coulomb interaction. For example, one effect is to push the charged lipids apart. By giving the tails more room, this increases the importance of the entropy and tends to destabilize the lamellar phase, just as an increase in temperature does. A second effect is that the charged headgroups also attract more counter ions, and these essentially increase the size of the headgroup which tends to stabilize the lamellar phase. There is clearly a competition between these two effects, one which depends upon the size of the counter ions. In the case of water, the lamellar phase is stabilized as the headgroups become more charged [30], as is observed experimentally [28].

In the future, one hopes to apply the model to other problems such as membrane fusion and to lipid protein interactions. Furthermore it would be of interest to treat the chains within the rotational isomeric states model [31] so that the effects of double bonds, their number and placement, on the self-assembly of lipids could be incorporated. Initial studies [32] show such an approach to be feasible.

This work has been supported by the National Science Foundation under grant number DMR9876864.

\section{References}

1. Semenov A.N. Contribution to the theory of microphase layering in block-copolymer melts. // Sov. Phys. JETP, 1985, vol. 61, p. 733-742.

2. Leibler L. Theory of microphase separation in block copolymers. // Macromolecules, 1980, vol. 13, p. $1602-1617$.

3. Thomas E.L., Alward D.B., Kinning D.J., Martin D.C., Handlin D.L. Jr., Fetters L.J. Ordered bicontinuous double diamond structure of star block copolymers: a new equilibrium morphology. // Macromolecules, 1986, vol. 19, p. 2197-2202.

4. Hamley I.W., Koppi K.A., Rosedale J.H., Bates F.S., Almdal K., Mortensen K. Hexagonal mesophases between lamellae and cylinders in a diblock copolymer melt. // Macromolecules, 1993, vol. 26, p. 5959-5970.

5. Hadjuk D.A., Harper P.E., Gruner S.M., Honeker C.C., Kim G., Thomas E.L., Fetters L.J. The gyroid: a new equilibrium morphology in weakly segregated diblock copolymers. // Macromolecules, 1994, vol. 27, p. 4063-4075.

6. Edwards S.F. The theory of polymer solutions at intermediate concentration. // Proc. Phys. Soc., 1966, vol. 88, p. 265-280.

7. Helfand E., Wasserman Z.R. Block Copolymer theory 4. Narrow interphase approximation. // Macromolecules, 1976, vol. 9, p. 879-888.

8. Matsen M.W., Schick M. Stable and unstable phases of a diblock copolymer melt. // Phys. Rev. Lett., 1994, vol. 72, p. 2660-2663.

9. Hadjuk D.A., Harper P.E., Gruner S.M., Honeker C.C., Thomas E.L., Fetters L.J. A reevaluation of bicontinuous cubic phases in starblock copolymers. // Macromolecules, 1995, vol. 28, p. 2570-2573. 
10. Hadjuk D.A., Takenouchi H., Hillmyer M.A., Bates F.S., Vigild M.E., Almdal K. Stability of the perforated (PL) phase in diblock copolymer melts. // Macromolecules, 1997, vol. 30, p. 3788-3795.

11. Matsen M.W., Bates F.S. Unifying weak- and strong-segregation block copolymer theories. // Macromolecules, 1996, vol. 29, p. 1091-1098.

12. Thomas E.L., Anderson D.M., Henkee C.S., Hoffman D. Periodic area-minimizing surfaces in block copolymers. // Nature, 1988, vol. 334, p. 598-601.

13. Matsen M.W., Bates F.S. Origins of complex self-assembly in block copolymers. // Macromolecules, 1996, vol. 29, p. 7641-7644.

14. Jinnai H., Nishikawa Y., Spontak R.J., Smith S.D., Agard D.A., Hashimoto T. Direct measurement of interfacial curvature distributions in a bicontinuous block copolymer morphology. // Phys. Rev. Lett., 2000, vol. 84, p. 518-521.

15. Matsen M.W. Thin films of block copolymer. // J. Chem. Phys., 1997, vol. 106, p. $7781-7791$.

16. Janert P.K., Schick M. Phase behaviour of ternary homopolymer/diblock blends: microphase unbinding in the symmetric system. // Macromolecules, 1997, vol. 30, p. 3916-3920.

17. Matsen M.W. Kink grain boundaries in a block copolymer lamellar phase. // J. Chem Phys, 1997, vol. 107, p. 8110-8119.

18. Duque D., Schick M. Self consistent field theory of twist grain boundaries in block copolymers. // J. Chem. Phys., 2000, vol. 113, p. 5525-5530.

19. Epand R.M. Current Topics in Membranes. Vol. 44. San Diego, Academic press. 1997.

20. Li X.-J., Schick M. Theory of lipid polymorphism: application to phosphatidylethanolmine and phosphatidylserine. // Biophys. J., 2000, vol. 78, p. 34-46.

21. Israelachvili J.N. Intermolecular and Surface Forces. San Diego, Academic press. 1985.

22. Gruner S.M. Stability of lyotropic phases with curved interfaces. // J. Phys. Chem., 1989, vol. 93, p. 7562-7570.

23. Gawrisch K., Parsegian V.A., Hadjuk D.A., Tate M.W., Gruner S.M., Fuller N.L., Rand P.R. Energetics of a hexagonal-lamellar-hexagonal-phase transition sequence in dioleoylphosphatidylethanolamine membranes. // Biochemistry, 1992, vol. 31, p. $2856-2864$.

24. Markin V.S., Kozlov M.M., Borovjagin V.L. On the theory of membrane fusion. The stalk mechanism. // Gen. Physiol. Biophys., 1984, vol. 5, p. 361-377.

25. Li X.-J., Schick M. Distribution of lipids in nonlamellar phases of their mixtures. // J. Chem. Phys., 2000, vol. 112, p. 6063-6072.

26. Li X.-J., Schick M. Fluctuations in mixtures of lamellar and nonlamellar-forming lipids. // J. Chem. Phys., 2000, vol. 112, p. 10599-10607.

27. Laradji M., Shi A.-C., Noolandi J., Desai R.C. Stability of ordered phases in diblock copolymer melts. // Macromolecules, 1997, vol. 30, p. 3242-3255.

28. Hope M.J., Cullis P.R. Effects of divalent cations and $\mathrm{pH}$ on phosphatidylserine model membranes: a ${ }^{31} \mathrm{P}$ NMR study. // Biochim. Biophys. Res. Comm., 1980, vol. 92, p. 846-852.

29. Hafez I.M., Ansell S., Cullis P.R. Tunable $\mathrm{pH}$ sensitive liposomes composed of mixtures of cationic and anionic lipids. // Biophys. J., 2000, vol. 79, p. 1438-1446.

30. Li X.-J., Schick M. Theory of tunable pH-sensitive vesicles of anionic and cationic lipids or anionic and neutral lipids. // Biophys. J., 2001, vol 80, p.1703-1711.

31. Flory P.J. Statistical Mechanics of Chain Molecules. New York, Wiley Interscience. 
1969.

32. Müller M., Schick M. Calculation of the phase behaviour of lipids. // Phys. Rev. B., 1998, vol. 57, p. 6973-6978.

\title{
Самоасоціація кополімерів та ліпідів
}

\author{
Х.-Дж. Лі, М.Шік
}

Університет Вашингтона, Сіетл 98195-1560, США

Отримано 14 серпня 2000 р.

Представлено огляд останніх досягнень у розумінні та обчисленні самоасоціації у блочних кополімерах і біологічних ліпідах (як у нейтральних, так і в заряджених).

Ключові слова: самоасоціація, ліотронні фази, блочні кополімери, ліпіди

PACS: $61.25 . H q, 87.15 .-v, 87.30 . H q$ 
